\title{
Curcumin induces mitochondria pathway mediated cell apoptosis in A549 lung adenocarcinoma cells
}

\author{
QING YONG CHEN ${ }^{1}$, GUO HUA LU ${ }^{2}$, YU QUAN WU ${ }^{1}$, YING ZHENG ${ }^{1}$, KEDI XU $^{3}$, \\ LI JUN WU ${ }^{1}$, ZHONG YONG JIANG ${ }^{1}$, RUI FENG ${ }^{1}$ and JIAN YING ZHOU ${ }^{2}$ \\ ${ }^{1}$ Department of Respiratory Disease, The 117th Hospital of PLA, Hangzhou, Zhejiang 310013; \\ ${ }^{2}$ Department of Respiratory Disease, First Affiliated Hospital, College of Medicine, Zhejiang University; \\ ${ }^{3}$ Department of Biomedical Engineering, Key laboratory for Biomedical Engineering of Ministry of Education, \\ Zhejiang University, Hangzhou, Zhejiang 310029, P.R. China
}

Received November 3, 2009; Accepted December 10, 2009

DOI: $10.3892 /$ or_00000762

\begin{abstract}
Several studies have shown that curcumin can induce apoptosis and inhibit growth in human tumor cell lines. However, the mechanism is not completely understood yet. The present studies were designed to investigate the effects of curcumin on human A549 lung adenocarcinoma cells lines to better understand its effect on apoptosis and apoptosis-related genes in vitro. Apoptosis induction, mitochondria membrane potential, mitochondria structure, and apoptotic associated gene expression were examined by flow cytometric assay, confocal microscopy, Western blotting and electron microscopy. After treatment with curcumin, percentage of apoptotic cells increased dose- and timedependently, and morphology observation revealed typical apoptotic features. Our data further indicated that the expression of Bax proteins in A549 cells was increased in a dose-dependent manner, whereas the expression of $\mathrm{Bcl}-2$ was significantly decreased, thus the ratio of Bax/Bcl-2 was increased. The apoptotic process was accompanied by the change of mitochondrial function and structure which led to release of the cytochrome $c$, and activation of caspase- 9 and
\end{abstract}

Correspondence to: Dr Qing Yong Chen, Department of Respiratory Disease, The 117th Hospital of PLA, 14 Lingying Road, Hangzhou, Zhejiang 310013, P.R. China

E-mail: cqyong117@163.com or cqyong117@hotmail.com

Dr Jian Ying Zhou, Department of Respiratory Diseases, The First Affiliated Hospital, College of Medicine, Zhejiang University, 79 Qingchun Road, Hangzhou, Zhejiang 310003, P.R. China

E-mail: drzjy@163.com

Abbreviations: JC-1, 5,5',6,6'-tetrachloro-1,1',3,3'-tetraethylbenzimidazolylcarbocyanineiodide; PI, propidium iodide; FBS, fetal bovine serum; DMSO, dimethylsulfoxide; PBS, phosphate-buffered saline; PARP, poly-ADP-ribose polymerase

Key words: curcumin, apoptosis, lung cancer, mitochondria membrane potential, caspases caspase-3. Furthermore, curcumin also induced a dosedependent cleavage of PARP. Caspases activation during the course of curcumin-induced apoptosis was additionally confirmed by using a broad-spectrum caspases inhibitor, ZVAD-fmk. As expected, the inhibitor was able to decrease curcumin-induced apoptosis on A549 cells. These results suggested that mitochondria played an important role in the curcumin-induced apoptosis, and mitochondria membrane potential loss initiated apoptosis via the activation of caspases.

\section{Introduction}

Curcumin-[1,7-bis-(4-hydroxy-3-methoxyphenyl)-1,6-heptadiene-3,5-dione(diferuloyl methane)], a natural and crystalline compound isolated from the plant Curcuma longa, has been recognized as a promising anticancer drug due to its multiple properties including anti-inflammatory, anti-oxidant and anticarcinogenic activities (1). Although the precise mode of action of this compound is not yet elucidated, Several studies have shown that curcumin induces apoptosis more potently in cancer cells than in normal cells $(2,3)$. Furthermore, selective anticancer activity of curcumin has been demonstrated for several types of tumor. The anticancer activity of curcumin has been attributed to the inhibition of cell proliferation, induction of apoptosis $(4,5)$, and inhibition of angiogenesis and metalloproteinases $(1,6)$. Modulation of several target genes have been implicated in the anticancer activity of curcumin, including inhibition of nitric oxide synthase, receptor tyrosine kinase and protein kinase $\mathrm{C}$ activities $(7,8)$ and the alteration of transcriptional factors c-jun/AP-1, nuclear factor $\kappa \mathrm{B}$ and p53 by curcumin have been suggested $(9,10)$. Recently it has also been suggested that production of reactive oxygen intermediates the release of cytochrome c causing tumor cell apoptosis as a result of curcumin treatment (11). Curcumin also has been reported to induce mitochondrial abnormalities and promote p53-dependent apoptosis and the activation of caspase- 8 and caspase-3 (12-16). However, little is known about the effect of curcumin at molecular level on human A549 lung adenocarcinoma cells.

To gain a better understanding of the effects of curcumin on lung cancer cells, the present investigation was designed 
to explore its molecular mechanism of action by induced apoptosis in A549 cells in vitro. Our results showed that curcumin was able to induce apoptosis in human A549 cells through caspase-dependent mitochondria pathway and the regulation of apoptosis-related proteins, which suggested that such a multi-marker analysis of apoptosis pathways, could be useful for individualization of therapeutic strategies.

\section{Materials and methods}

Regants and cells culture. Curcumin, annexin V-FITC and propidium iodide (PI) were purchased from Sigma Chemical (St. Louis, MO, USA). Fetal bovine serum (FBS), RPMI-1640, and penicillin-streptomycin were obtained from Gibco-BRL (Gaithersburg, MD, USA). Caspase-3, PARP, caspase-9, Bcl-2, Bax and cytochrome $\mathrm{c}$ antibodies were purchased from Santa Cruz Biotechnology, USA. 5,5',6,6'-Tetrachloro1,1',3,3'-tetraethylbenzimidazolylcarbo-cyanine iodide (JC-1), and Mitotracker Red CMXRos were purchased from Molecular Probes, Inc. (Eugene, OR, USA). Human A549 lung carcinoma cells were cultured using previously described methods (17).

Evaluation of apoptosis by flow cytometry. For detection of apoptosis by FACS, A549 cells were treated with different concentrations of curcumin. Harvested cells were doublestained with FITC-conjugated Annexin V and PI for 5 min at room temperature as previously described (17).

Electron microscopy. A549 cells treated with $20 \mu \mathrm{M}$ curcumin for $24 \mathrm{~h}$ were fixed in $2.0 \%$ glutaraldehyde in $0.1 \mathrm{M}$ cacodylate buffer ( $\mathrm{pH} 7.4$ ) supplemented with $0.1 \mathrm{M}$ sucrose (Cac buffer) for $6 \mathrm{~h}$, rinsed in Cac buffer and post-fixed for $1.5 \mathrm{~h}$. Subsequently, the cells were dehydrated in ethanol, immersed in propylene oxide and embedded in epoxy resin (Agar 100, Agar Scientific, Stansted, UK). Ultra thin sections were contrasted with $4 \%$ uranyl acetate in water for $30 \mathrm{~min}$ at $20^{\circ} \mathrm{C}$ and Reynolds lead citrate for $5 \mathrm{~min}$ at $20^{\circ} \mathrm{C}$ before examination under an electron microscope.

Western blot analysis. Cells were lysed in a lysis buffer $(50 \mathrm{mM}$ Tris-HCl, pH 7.8, $150 \mathrm{mM} \mathrm{NaCl}, 1 \%$ NP40, 0.1\% SDS, 1 mM phenylmethylsulfonyl fluoride). The lysates were resolved on SDS-PAGE gels, and then transferred to polyvinylidine difluoride membranes as previously described (17).

Measurement of the change of mitochondrial membrane potential. Changes of mitochondrial membrane potential were monitored by determination of the fluorescence of the lipophilic cationic probe JC-1. Cells were treated with or without curcumin for the indicated time periods. Thirty minutes before the termination of incubation, JC-1 dye (final concentration $40 \mu \mathrm{M}$ ) was added to the media and incubated for $30 \mathrm{~min}$ at $37^{\circ} \mathrm{C}$ in the dark. The cells were finally harvested and re-suspended in PBS. The accumulation of JC- 1 was analyzed immediately by flow cytometry or confocal microscopy.

Immunofluorescence analysis. Cells were incubated for $30 \mathrm{~min}$ in normal growth medium containing $10 \mathrm{nM}$ Mitotracker Red CMXRos. Thereafter, cells were cyto-spinned on glass slides,
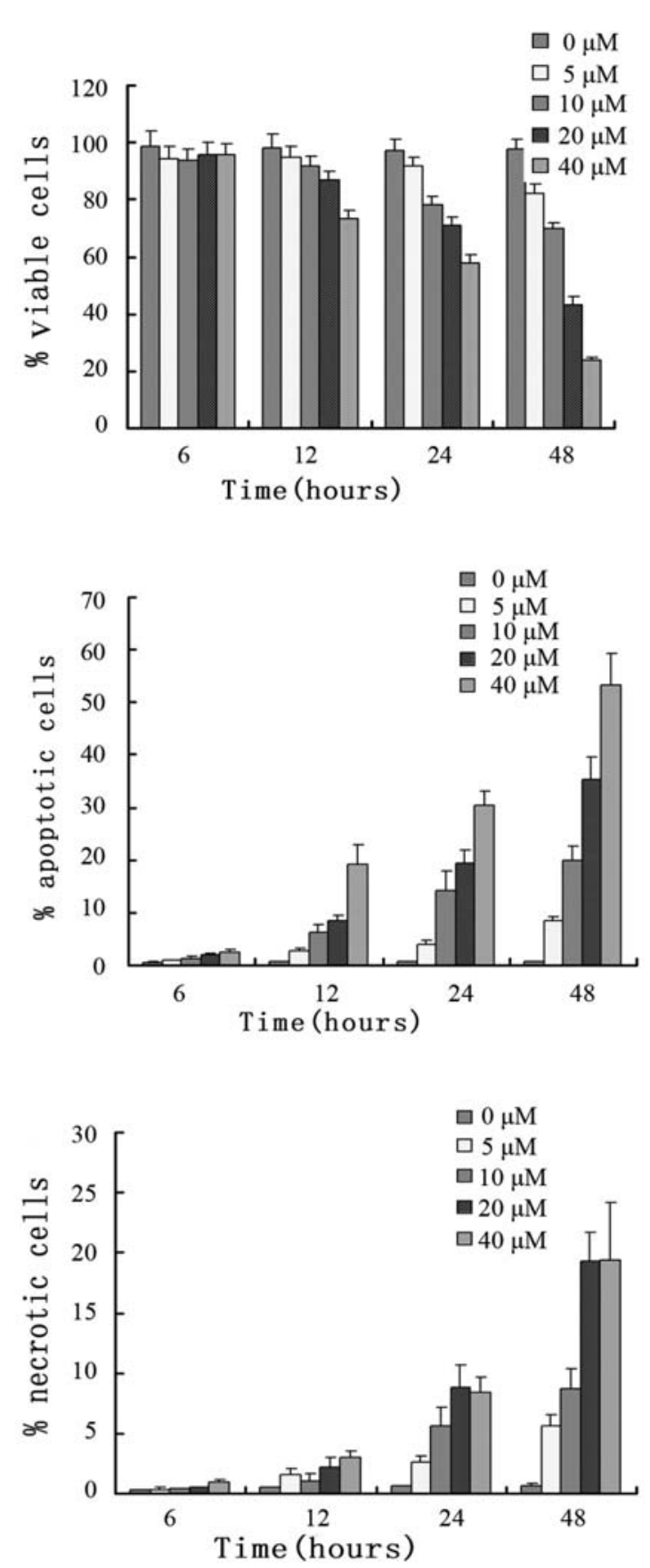

Figure 1. Apoptotic cells detected by flow cytometry with Annexin V conjugated with PI staining. A549 cells were treated without curcumin or with curcumin at different concentrations for 6-48 h. The assay was performed according to the Materials and methods. Viable cells, apoptotic cells, and necrotic cells were analyzed.

and washed once with PBS, fixed with $4 \%$ paraformaldehyde for 20 min, permeabilized with $0.2 \%$ Triton $\mathrm{X}-100$ during $10 \mathrm{~min}$ and incubated with phosphate-buffered saline (PBS) containing 4\% BSA for $1 \mathrm{~h}$ at room temperature. Primary antibodies diluted in PBS containing 1\% BSA were used in a dilution of 1/200 for anti-Bax and 1/100 for anticytochrome $\mathrm{c}$ and incubated for $1 \mathrm{~h}$ at room temperature. Afterwards, antigen-antibody complex was washed and incubated with FITC conjugated secondary antibody. Following the staining cells were washed twice in PBS. Fluorescence images were collected on a Zeiss Axioplan 

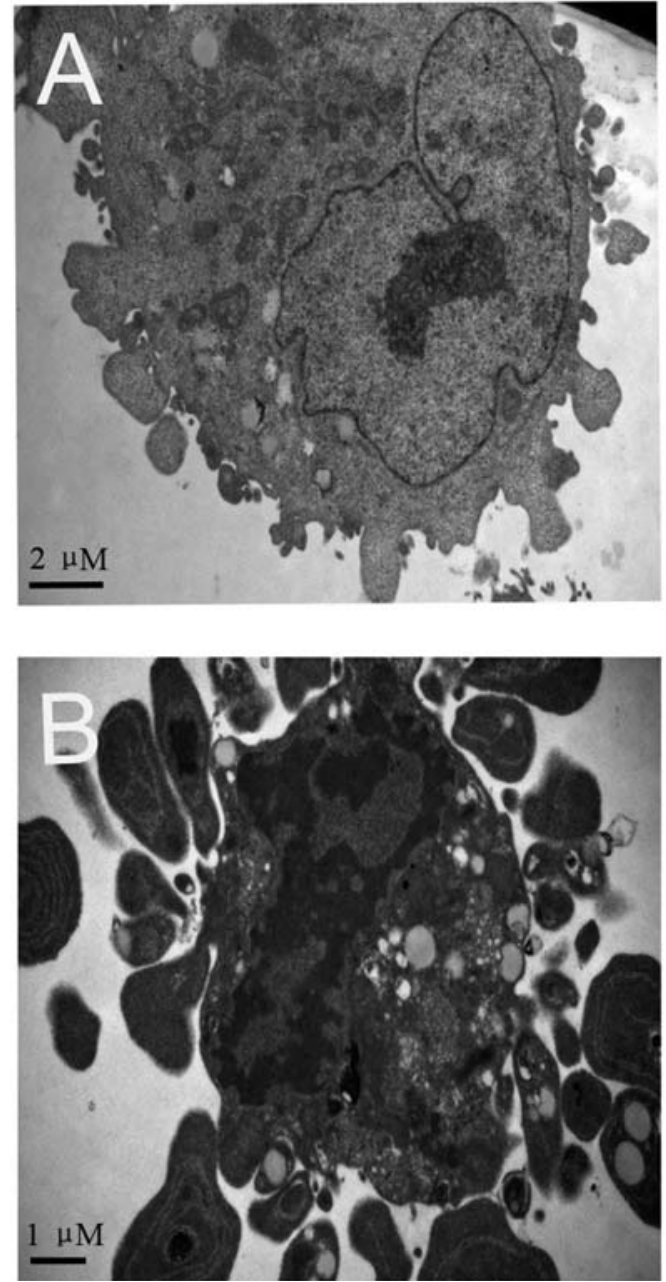

Figure 2. Effect of curcumin on the cellular ultrastructure. Cells treated with $20 \mu \mathrm{M}$ curcumin for $24 \mathrm{~h}$ were fixed with $2 \%$ glutaraldehyde for $15 \mathrm{~min}$ Following several rinses in PBS, cells were post-fixed and dehydrated as described in Materials and methods. (A) Control A549 cells exposed to $0.1 \%$ DMSO. (B) A549 cells exposed to curcumin $(20 \mu \mathrm{M})$.

microscope and 10 sections of each preparation were scanned.

Statistical analysis. All data are presented as means \pm SD with $n=3$ or more and independent experiments were repeated at least 3 times. Student's t-test was used to determine the significance of statistical differences between data at the level of $\mathrm{P}<0.05$.

\section{Results}

Curcumin induced apoptosis of A549 cells. To determine whether curcumin induces apoptosis of A549 cells, A549 cells were exposed to various concentrations of curcumin (5-40 $\mu \mathrm{M})$, harvested at different time-points, and apoptosis was investigated using Annexin V-FITC/PI assay. As shown in Fig. 1, when cells were exposed to curcumin for $24 \mathrm{~h}$, the proportion of $\mathrm{AV}^{+} / \mathrm{PI}^{+}$(necrotic cells) was increased from $0.59 \%$ of control cells to $8.79 \%$ of the $40 \mu \mathrm{M}$ curcumin group, and the number of $\mathrm{AV}^{+} / \mathrm{PI}^{-}$(apoptotic cells) increased from 0.66 to $30.49 \%$. After $48 \mathrm{~h}$ treatment, the proportion of necrotic cells was increased from $0.62 \%$ of control cells to $19.42 \%$ of the $40 \mu \mathrm{M}$ curcumin group, and apoptotic cells
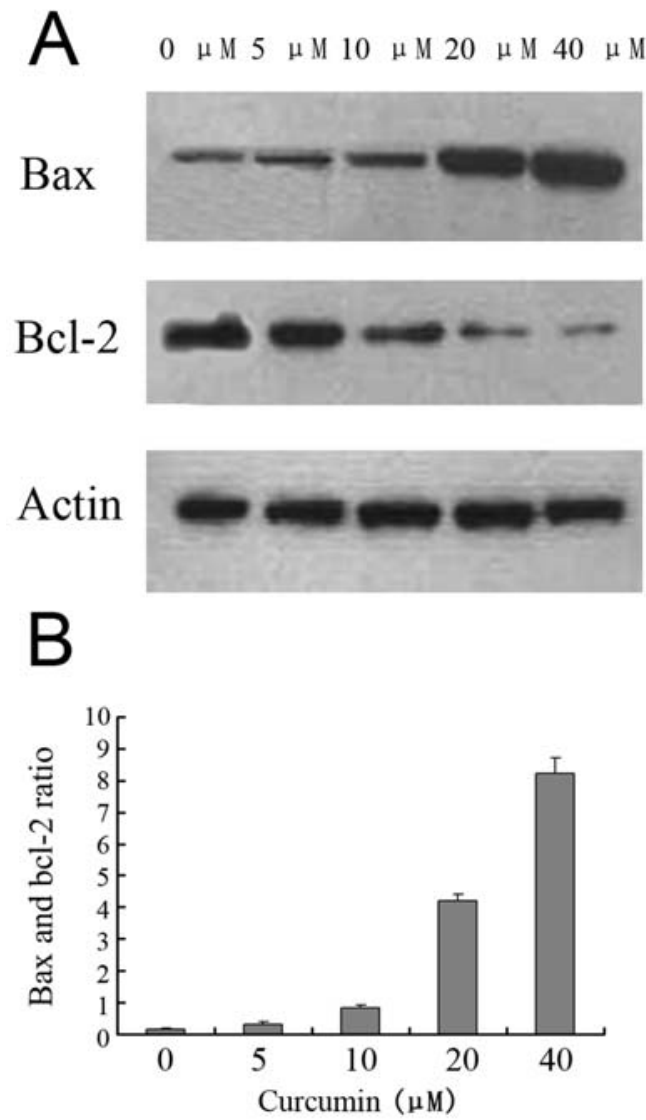

Figure 3. Effects of curcumin on the expression of Bcl-2 family of proteins A549 cells were treated with or without curcumin $(5-40 \mu \mathrm{M})$ for $24 \mathrm{~h}$, and then cells were harvested and prepared for the detection of protein expression of Bax and Bcl-2 using Western blots. Densitometric analysis shows: Bax, Bcl-2 ratio. Data are representative of three independent experiments.

were significantly elevated in curcumin treated cells from $0.71 \%$ in untreated cells to $53.21 \%$ after treatment with $40 \mu \mathrm{M}$ curcumin. The number of AV-/PI- (viable cells) decreased from 97.52 to $23.62 \%$. These results suggested that both necrosis and apoptosis contributed to the curcumin-induced death of A549 cells and curcumin-induced apoptosis was the main cause.

Morphological features of apoptosis. We next examined the cell morphologic characteristics by electron microscope. The results found that curcumin treated A549 cells showed morphological changes characteristics of apoptosis, including disappearance of microvilli, cell shrinkage, chromatin condensation containing a half-moon of condensed chromatin and appearance of membrance blebbing and many apoptotic bodies when compared with untreated cells (Fig. 2).

Effect of curcumin on the expression of the Bcl-2 family of proteins. There are several lines of evidence suggesting that Bcl-2 family of proteins plays a crucial role in mitochondriamediated apoptosis, we next examined the effect of curcumin on the constitutive levels of several Bcl-2 family protein members. The data showed that curcumin modified the level of Bax and Bcl-2 expression, induced a profound increase of Bax protein and decrease of $\mathrm{Bcl}-2$ protein, thus the ratio of Bax/Bcl-2 was increased (Fig. 3). Furthermore, the trans- 


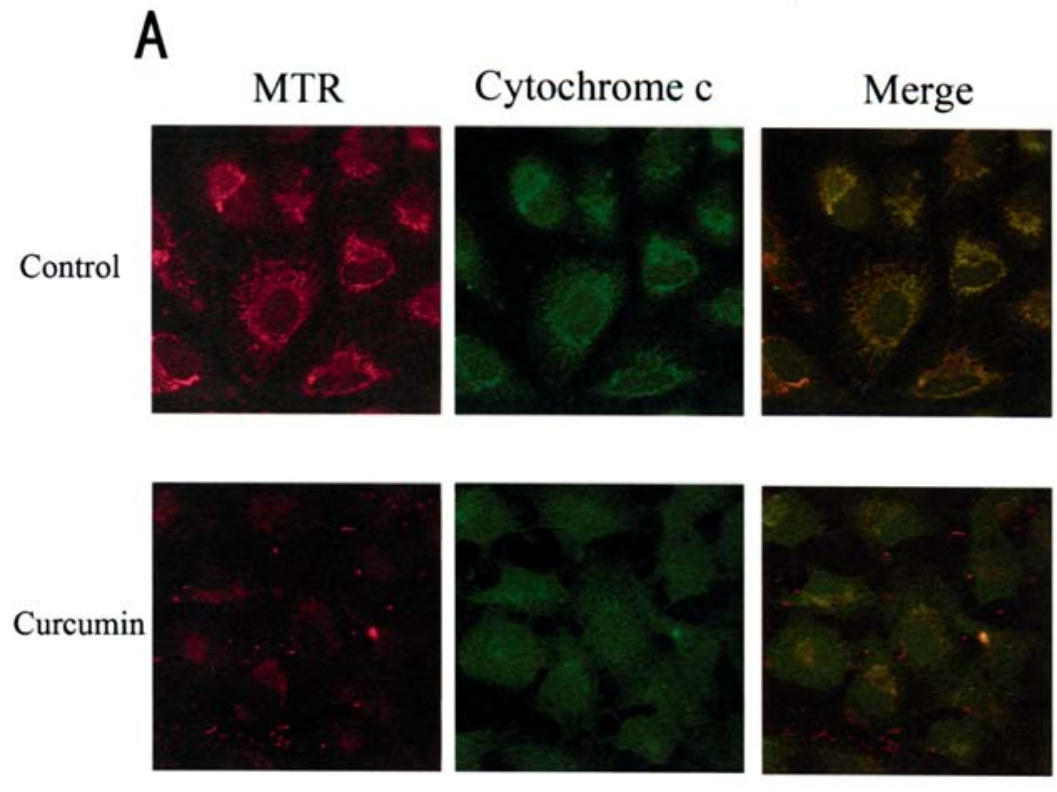

B
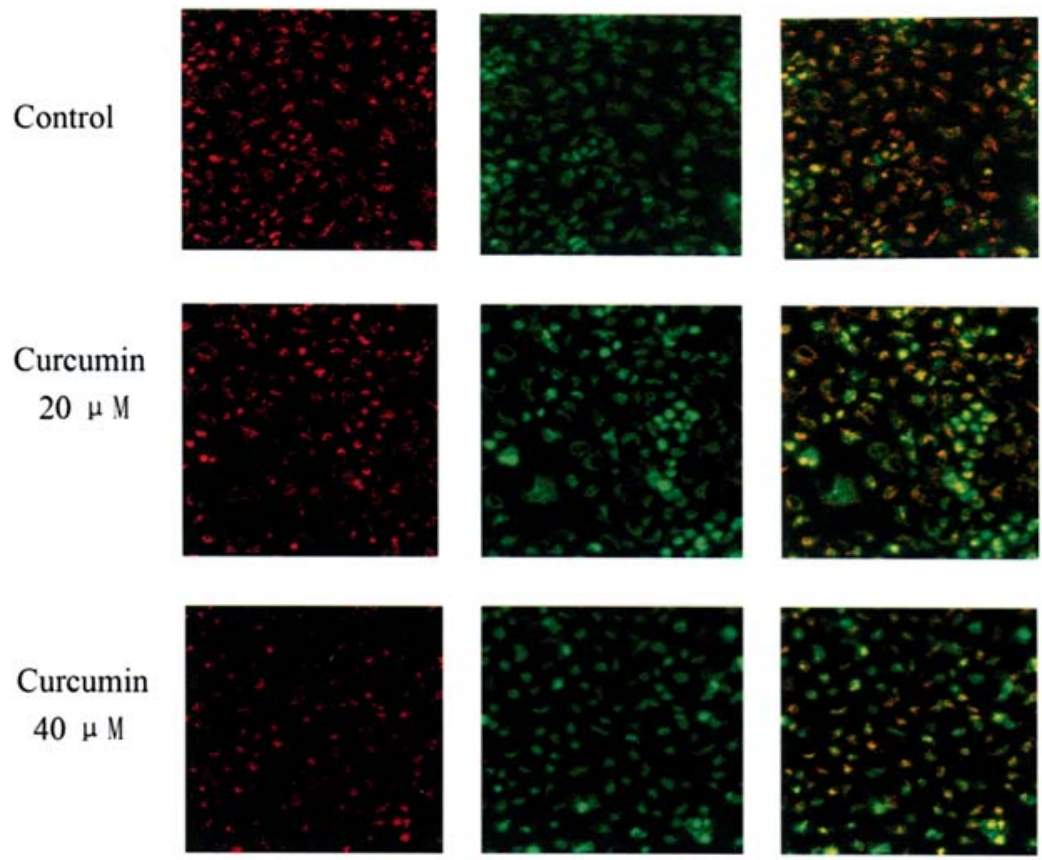

Figure 4. A and B, Effect of curcumin on the mitochondria pathway.

location of Bax from the cytosol into mitochondria has been reported to facilitate the release of mitochondria intermembrane proteins following induction of apoptosis (18). Accordingly, we monitored the localization of endogenous Bax in A549 cells. Indirect immunofluorescence staining revealed that the Bax protein is predominantly distributed in the cytosol under control conditions, but is no dramatically translocated into the mitochondria of cells after treated with curcumin (data no shown). These results showed that the increase in $\mathrm{Bax} / \mathrm{Bcl}-2$ ratio due to curcumin treatments indicated involvement of mitochondrial events in A549 cells for apoptosis.
Curcumin caused release of cytochrome c from mitochondria. Cytochrome $\mathrm{c}$ is localized in mitochondrial inter-membrane space and released into the cytosol upon apoptotic stimulation due to collapse of mitochondrial membrane potential. Upon release from mitochondria to the cytosol, cytochrome c complexes with procaspase-9 and Apaf - 1 to form the apoptosome, which initiates cleavage and activation of initiator (e.g., caspase-9) and executioner caspases (e.g., caspase-3). Therefore, we examined the cytochrome c levels by indirect immunofluorescence staining. In DMSO-treated control A549 cells, cytochrome c was mainly localized in the mitochondria as evidenced by yellow-orange staining due to 

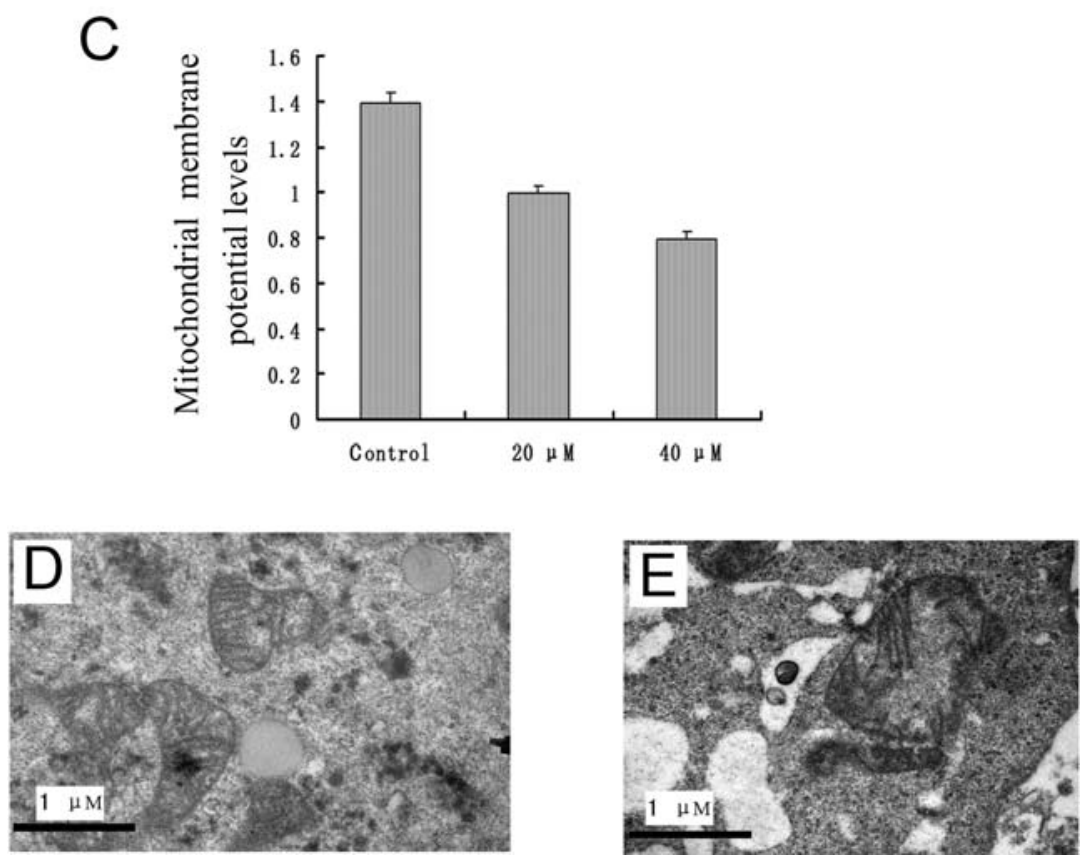

Figure 4. Effect of curcumin on the mitochondria pathway. After $24 \mathrm{~h}$ of culturing, the control and curcumin treated cells were incubated for $30 \mathrm{~min}$ with $10 \mathrm{~nm}$ Mitotracker (red), and then cells were cytospinned on glass slides, fixed and stained with cytochrome c antibody (green). The slides were analyzed by a confocal laser scanning microscope (A). Cells were stained with $10 \mu \mathrm{g} / \mathrm{ml}$ of JC-1 and observed under confocal microscope (B) and flow cytometry (C). Representative transmission electron micrographs of A549 cells following a 24-h treatment with DMSO (D) or $20 \mu \mathrm{M}$ curcumin (E).

merging of red (Mitotracker Red) and cytochrome cassociated green fluorescence. On the other hand, the yelloworange staining was markedly abolished in A549 cells upon treatment with curcumin. Instead, a large fraction of curcumin-treated A549 cells exhibited green fluorescence indicating release of cytochrome $\mathrm{c}$ from mitochondria into the cytosol. These data revealed that a treatment of A549 cells with curcumin increased cytochrome c level in the cytosol due to a concomitant decreased cytochrome c level in the mitochondria (Fig. 4A).

Curcumin induces apoptosis is through a mitochondriadependent pathway. Mitochondria play a major role in apoptosis triggered by many stimuli. The early loss of mitochondrial membrane potential is a hallmark of apoptosis. We examined the effect of curcumin on mitochondrial membrane potential by means of the sensitive dye JC-1. Confocal microscopy showed a dose-dependent increase in cells with green fluorescence (from JC-1 dye in the cytosol) as compared with red fluorescence (from JC-1 dye in the mitochondria) after a 24-h treatment of curcumin (Fig. 4B). We next quantified the extent of mitochondrial membrane potential by flow cytometric analysis of curcumin-treated cells. Consistent with the confocal microscopy data, flow cytometry revealed that treatment of A549 cells with curcumin resulted in a dose-dependent reduction of mitochondrial membrane potential (Fig. 4C). In addition, electron microscopy was used to examine the mitochondrial features of A549 cells treated with the vehicle DMSO or $20 \mu \mathrm{M}$ curcumin for $24 \mathrm{~h}$. The mitochondria in a DMSO-treated cell appeared to be electron dense with condensed, well-organized cristae (Fig. 4D). Conversely, the mitochondria in the curcumintreated cells looked abnormally enlarged or swollen, revealing
A

\section{Pro-caspase 9 cleaved form}

B

Pro-caspase 3
cleaved form

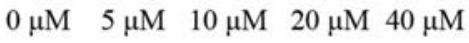
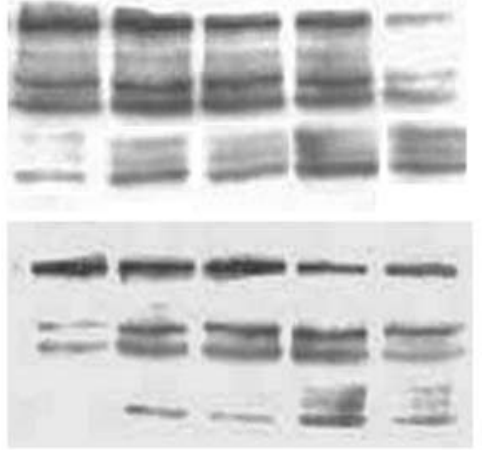

$\mathrm{C}$

PARP
cleaved form
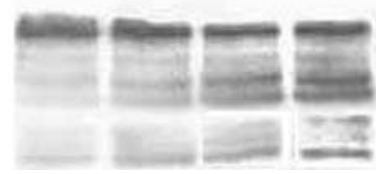

Figure 5. Effect of curcumin on the caspase activation in A549 cells. Cells were incubated in the absence or presence of curcumin $(5-40 \mu \mathrm{M})$ for $24 \mathrm{~h}$. Then, the cells were harvested and lysed for the detection of protein expressions of caspase-9 (A), caspase-3 (B) and PARP cleavage (C) by Western blot analysis.

shredded cristae and the disruption of outer membrane integrity (Fig. 4E). The data demonstrated that curcumin induced a lost of mitochondrial membrane potential or a visible change in mitochondrial morphology that correlates well with the apoptotic cell death.

Involvement of caspases activation in curcumin-induced apoptotic effect. The activation of caspases is a critical event 

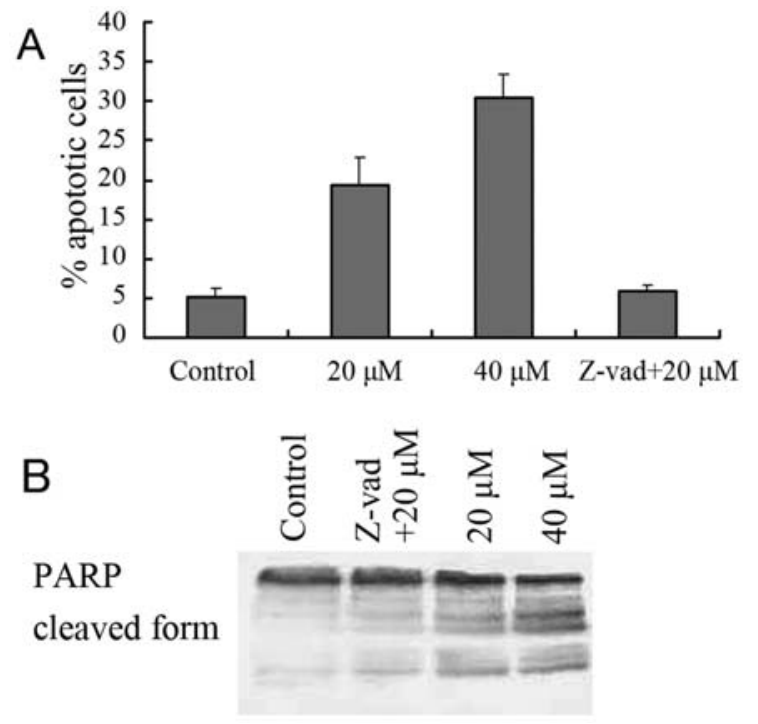

Figure 6. Effect of Z-VAD-fmk on curcumin-induced apoptosis. A549 cells were treated with $20 \mu \mathrm{M}$ curcumin in the presence or absence of caspase inhibitors Z-VAD-fmk $(50 \mu \mathrm{M})$. After 24-h incubation, cells were analyzed to examine cell death by flow cytometry (A) and PARP cleavage detected by Western blotting (B).

in the proteolytic cascade elicited by apoptotic stimuli; in particular caspase- 3 is an effector caspase that plays a role in cell death induced by a variety of stimuli. We further investigated the involvement of caspases activation in the curcumininduced apoptosis in the A549 cells by Western blot analyses. Consistent with an induction of apoptosis, we found that caspase- 3 and caspase- 9 were activated in curcumin-treated A549 cells (Fig. 5A, B) in a dose-dependent manner. During the process of apoptosis, poly-ADP-ribose polymerase (PARP) is inactivated due to specific cleavage of the protein into two fragments. The active form of caspase- 3 is responsible for the cleavage of PARP through the recognition of a specific site. Thus, it was of interest to examine whether curcumin was also capable of inducing cleavage of PARP. As expected, treatment of A549 cells with curcumin results in a dosedependent cleavage of PARP (Fig. 5C).

Previous studies have shown that curcumin exposure led to the activation of caspases at the initiation and the execution phase (19), thus we tested whether the change in mitochondrial membrane potential was tied to the processes in the caspase-dependent pathway. The results showed that $50 \mu \mathrm{M} \mathrm{Z}$-VAD-fmk did not affect the curcumin-induced reduction of mitochondrial membrane potential and release of cytochrome c (data no shown). Under the same experimental conditions, we found that curcumin-induced PARP protein cleavage and cell death were significantly inhibited by $50 \mu \mathrm{M}$ Z-VAD-fmk (Fig. 6). These data suggested that the disruption of mitochondrial functions was not through the caspases-dependent pathway, and the change in mitochondrial membrane potential comprised an upstream event resulting from caspase activity in the curcumin-induced apoptosis.

\section{Discussion}

Apoptosis results from the action of a genetically encoded suicide program that leads to cellular, morphological, and biochemical changes that include cell volume loss, mitochondrial depolarization, caspases activation, chromatin condensation and nuclear fragment. In the present study, curcumin remarkably induced lung cancer A549 cell apoptosis in dose- and time-dependent manner, which was confirmed by PI and Annexin V staining. The morphological changes characteristic of apoptotic cell death was also investigated by electron microscopy. As evidence of apoptosis, the morphologic changes characteristic of apoptosis nuclei of A549 cells incubated with $20 \mu \mathrm{M}$ curcumin was the disappearance of microvilli, cell shrinkage, nuclear fragments containing a half-moon of condensed chromatin and appearance of membrance blebbing when compared with untreated cells.

The anti-apoptotic proteins (e.g., Bcl-2 and Bcl-xL) prevent cytochrome c release by forming heterodimer complexes with pro-apoptotic Bcl-2 family proteins (e.g., Bax and Bak). The pro-apoptotic Bcl-2 proteins including Bax and Bak facilitate release of apoptogenic molecules from mitochondria to the cytosol $(20,21)$. To investigate the mitochondrial apoptotic events involved in curcumin-induced apoptosis, we first analyzed the changes in the levels of proapoptotic proteins Bax and anti-apoptotic proteins Bcl-2. Immunoblot analysis showed that treatment of A549 cells with curcumin increased Bax protein levels. In contrast, curcumin decreased Bcl-2 levels, which led to an increase in the pro-apoptotic/anti-apoptotic $\mathrm{Bcl}-2$ ratio. However, some studies found that Bax protein is translocated to mitochondria from the cytoplasm and then insert itself into the outer mitochondrial membrane, permeabilizing the membrane, triggering the release of mitochondria-related apoptotic factors, and inducing cell apoptosis $(22,23)$. In this study, our indirect immunofluorescence staining analysis showed that Bax protein is no translocated to mitochondria from the cytoplasm, suggesting that curcumin induced cytochrome $\mathrm{c}$ release may occur by a mechanism independent of Bax translocation to the mitochondria. Our current observation is well-correlated with the earlier studies demonstrating that curcumin induced apoptosis due to increased Bax and decreased Bcl-2 in other tumor cells (24-26). The increase in $\mathrm{Bax} / \mathrm{Bcl}-2$ ratio indicated a commitment of the A549 cells to apoptosis via the mitochondria-dependent pathway.

Furthermore, it has been shown that the increase in Bax/Bcl-2 ratio caused caspase activation, a decline of mitochondrial membrane potential and subsequent cytochrome c release $(24,27)$. Mitochondrial cytochrome c release is a well-known pre-condition for formation of the apoptosome and activation of caspases for apoptosis $(28,29)$. Our data showed that curcumin induced increase in Bax/Bcl-2 ratio due to curcumin treatments concomitant with release of cytochrome $\mathrm{c}$ from mitochondria to the cytosol as evidenced by immunofluorescence studies. These data are in agreement with previous studies where curcumin induced mitochondrial release of cytochrome c leading to apoptosis in other tumor cells $(25,30,31)$. Thus, it seems that curcumin decreases mitochondrial membrane potential, at least in part, through a Bcl-2 family-mediated pathway.

Because curcumin treatment caused release of cytochrome c from mitochondria into the cytosol, we proceeded to determine its effect on mitochondrial membrane potential 
using a mitochondrial-specific cationic dye JC-1 to confirm loss of mitochondrial membrane potential by curcumin exposure. The JC-1 dye bearing a delocalized positive charge enters the mitochondrial matrix due to the negative charge established by the intact mitochondrial membrane potential. In cells with intact mitochondrial membrane potential, JC-1 predominantly exhibits red fluorescence due to formation of J-aggregates. In cells with mitochondrial membrane potential disruption, JC-1 dye accumulates in the cytoplasm in monomeric form (green fluorescence). In the control group, A549 cells predominantly exhibited red fluorescence indicating intact mitochondrial membrane potential. However, in curcumin-treated groups, A549 cells predominantly exhibited green fluorescence indicating loss of mitochondrial membrane potential. Consistent with the confocal microscopy data, flow cytometry revealed that treatment of A549 cells with curcumin resulted in a dose-dependent reduction of mitochondrial membrane potential. Because cytochrome c release from mitochondria may be involved in the opening of the permeability transition pore of the inner mitochondrial membrane. This opening should result in swelling of the mitochondria and the disruption of the outer mitochondrial membrane. Thus, the reduction of mitochondrial membrane potential by curcumin treatment evoked the notion of mitochondrial structure disruption in these cells. Electron microscopy was used to examine the mitochondrial features of A549 cells treated with the vehicle DMSO or $20 \mu \mathrm{M}$ curcumin for $24 \mathrm{~h}$. The mitochondria in a DMSO-treated cell appeared to be electron dense with condensed, well-organized cristae. Conversely, the mitochondria in the curcumin-treated cells looked abnormally enlarged or swollen, revealing shredded cristae and the disruption of outer membrane integrity. These results suggest that the decrease of mitochondrial membrane potential and increase of cytochrome c release may be due to the alteration in the mitochondria structure. In addition, the mitochondrial swelling and disruption have also been reported in various types of tumor cells exposed to other putative cancer chemopreventive agents during apoptosis $(32,33)$, suggesting that the change of mitochondrial function and structure observed during curcumin-induced apoptosis in A549 cells was perhaps not an agent- or cell type-specific phenomenon.

There is accumulating evidence indicating that mitochondria play a pivotal role in the apoptotic process in mammalian cells. Disruption of mitochondrial membrane potential is considered to be an indicator of mitochondria damage and generally is defined as an early stage of apoptosis, preceding efflux of small molecules from the mitochondria (including cytochrome c, apoptosis-inducing factor, cIAPs) and followed by caspase-9/caspase-3 cascade activation (34). Previous reports indicated that curcumin induced apoptosis is through a mitochondria-dependent activation of caspase- 3 and -9 in human in primary effusion lymphoma (35) and in human prostate cancer (31). In the present study, our results showed that the increased caspase- 3 and caspase- 9 activity in curcumin-treated cells is accompanied by cleavage of PARP. Furthermore, pretreatment of A549 cells with $50 \mu \mathrm{M}$ of Z-VAD-fmk, a universal inhibitor of caspases, prevents the cleavage of PARP and rescues cells from curcumin-induced apoptosis, clearly indicating that caspases play a critical role in curcumin induced apoptosis in A549 cells. However, ZVAD-fmk did not affect the curcumin-induced reduction of mitochondrial membrane potential and release of cytochrome c. These data showed that the activation of caspases depended on mitochondrial cytochrome c release to cytosol, and that it follows the change of mitochondrial function and structure, suggesting that mitochondria-mediated pathway is involved in curcumin-triggered apoptosis in A549 cells. This observation is consistent with the conclusion of a recent study on the effect of curcumin in human leukemia HL-60 cells (36).

In conclusion, the present study reveals that curcumin treatment causes apoptosis in A549 cells is associated with a change in the levels of Bcl-2 family proteins and release of cytochrome c concomitant with lost of mitochondrial membrane potential or a visible change in mitochondrial morphology; and curcumin induced cell death in our model is likely caused by activation of intrinsic caspase pathways. The ability of curcumin to induce apoptosis in lung cancer and other cancer cells indicates the possibility of developing curcumin as a potential cancer chemoprevention and chemotherapeutic agent.

\section{Acknowledgements}

This study was supported by the Zhejiang Medicine Administration Bureau (Project: N0.2008CA077) and the Funds of Nanjing Command Health Speciality ' 122 ' Engineering (Funds: N0.342510001). We would like to thank Dr Yakun Ge and Dr Lisheng Qian for their technical assistance and discussion.

\section{References}

1. Aggarwal BB, Kumar A and Bharti AC: Anticancer potential of curcumin: preclinical and clinical studies. Anticancer Res 23: 363-398, 2003.

2. Han SS, Chung ST, Robertson DA, Ranjan D and Bondada S: Curcumin causes the growth arrest and apoptosis of B cell lymphoma by down-regulation of egr-1, c-myc, bcl-XL, NFkappa B and p53. Clin Immunol 93: 152-161, 1999.

3. Ramachandran C and You W: Differential sensitivity of human mammary epithelial and breast carcinoma cell lines to curcumin. Breast Cancer Res Treat 54: 269-278, 1999.

4. Kuo ML, Huang TS and Lin JK: Curcumin, an antioxidant and anti-tumor promoter, induces apoptosis in human leukemia cells. Biochim Biophys Acta 1317: 95-100, 1996.

5. Mehta K, Pantazis P, McQueen T and Aggarwal BB: Antiproliferative effect of curcumin (diferuloylmethane) against human breast tumor cell lines. Anticancer Drugs 8: 470-481, 1997.

6. Li L, Braiteh FS and Kurzrock R: Liposome-encapsulated curcumin: in vitro and in vivo effects on proliferation, apoptosis, signaling and angiogenesis. Cancer 104: 1322-1331, 2005.

7. Pan MH, Lin-Shiau SY and Lin JK: Comparative studies on the suppression of nitric oxide synthase by curcumin and its hydrogenated metabolites through down-regulation of IkappaB kinase and NFkappaB activation in macrophages. Biochem Pharmacol 60: 1665-1676, 2000

8. Korutla L and Kumar R: Inhibitory effect of curcumin on epidermal growth factor receptor kinase activity in A431 cells. Biochim Biophys Acta 1224: 597-600, 1994.

9. Singh S and Aggarwal BB: Activation of transcription factor NF-kappa B is suppressed by curcumin (diferuloylmethane). J Biol Chem 270: 24995-25000, 1995. 
10. Jiang MC, Yang-Yen HF, Lin JK and Yen JJ: Differential regulation of $\mathrm{p} 53, \mathrm{c}-\mathrm{Myc}, \mathrm{Bcl}-2$ and $\mathrm{Bax}$ protein expression during apoptosis induced by widely divergent stimuli in human hepatoblastoma cells. Oncogene 13: 609-616, 1996.

11. Bhaumik S, Anjum R, Rangaraj N, Pardhasaradhi BV and Khar A: Curcumin mediated apoptosis in AK-5 tumor cells involves the production of reactive oxygen intermediates. FEBS Lett 456: 311-314, 1999

12. Mukhopadhyay A, Bueso-Ramos C, Chatterjee D, Pantazis P and Aggarwal BB: Curcumin down-regulates cell survival mechanisms in human prostate cancer cell lines. Oncogene 20 7597-7609, 2001.

13. Anto RJ, Mukhopadhyay A, Denning K and Aggarwal BB: Curcumin (diferuloylmethane) induces apoptosis through activation of caspase-8, BID cleavage and cytochrome c release: its suppression by ectopic expression of $\mathrm{Bcl}-2$ and $\mathrm{Bcl}-\mathrm{xl}$. Carcinogenesis 23: 143-150, 2002.

14. Morin D, Barthelemy S, Zini R, Labidalle S and Tillement JP: Curcumin induces the mitochondrial permeability transition pore mediated by membrane protein thiol oxidation. FEBS Lett 495: 131-136, 2001

15. Jee SH, Shen SC, Tseng CR, Chiu HC and Kuo ML: Curcumin induces a p53-dependent apoptosis in human basal cell carcinoma cells. J Invest Dermatol 111: 656-661, 1998.

16. Bush JA, Cheung KJ Jr and Li G: Curcumin induces apoptosis in human melanoma cells through a Fas receptor/caspase-8 pathway independent of p53. Exp Cell Res 271: 305-314, 2001.

17. Qing YC, Li JW, Yu Q W, Guo HL, Zhong YJ, Jian WZ, Yan J and Jian YZ: Molecular mechanism of trifluoperazine induces apoptosis in human A549 lung adenocarcinoma cell lines. Mol Med Rep 2: 811-817, 2009.

18. Jia L, Patwari Y, Srinivasula SM, Newland AC, Alnemri TF, Alnemri ES and Kelsey SM: Bax translocation is crucial for the sensitivity of leukaemic cells to etoposide-induced apoptosis. Oncogene 20: 4817-4826, 2001.

19. Holland JF, Elliott PJ, Adams J, Muggia FM and Perez-Soler R: Mechanisms of proteasome inhibitor PS-341-induced G(2)-Mphase arrest and apoptosis in human non-small cell lung cancer cell lines. Clin Cancer Res 9: 1145-1154, 2003.

20. Chao DT and Korsmeyer SJ: BCL-2 family: regulators of cell death. Ann Rev Immunol 16: 395-419, 1998.

21. Adams JM and Cory S: The Bcl-2 protein family: arbiters of cell survival. Science 281: 1322-1326, 1998.

22. Hsu YT, Wolter KG and Youle RJ: Cytosol-to-membrane redistribution of Bax and Bcl-X(L) during apoptosis. Proc Natl Acad Sci USA 94: 3668-3672, 1997.

23. Nomura M, Shimizu S, Ito T, Narita M, Matsuda $H$ and Tsujimoto Y: Apoptotic cytosol facilitates Bax translocation to mitochondria that involves cytosolic factor regulated by Bcl-2. Cancer Res 59: 5542-5548, 1999.
24. Sen S, Sharma H and Singh N: Curcumin enhances Vinorelbine mediated apoptosis in NSCLC cells by the mitochondrial pathway. Biochem Biophys Res Commun 331: 1245-1252, 2005.

25. Shi M, Cai Q, Yao L, Mao Y, Ming Y and Ouyang G: Antiproliferation and apoptosis induced by curcumin in human ovarian cancer cells. Cell Biol Int 30: 221-226, 2006.

26. Shankar S and Srivastava RK: Involvement of Bcl-2 family members, phosphatidylinositol 3'-kinase/AKT and mitochondrial p53 in curcumin (diferulolylmethane)-induced apoptosis in prostate cancer. Int J Oncol 4: 905-918, 2007.

27. Karmakar S, Banik NL, Patel SJ and Ray SK: Curcumin activated both receptor-mediated and mitochondria-mediated proteolytic pathways for apoptosis in human glioblastoma T98G cells. Neurosci Lett 407: 53-58, 2006.

28. Li P, Nijhawan D, Budihardjo I, Srinivasula SM, Ahmad M, Alnemri ES and Wang X: Cytochrome c and dATP-dependent formation of Apaf-1/caspase-9 complex initiates an apoptotic protease cascade. Cell 91: 479-489, 1997

29. Kluck RM, Bossy-Wetzel E, Green DR and Newmeyer DD: The release of cytochrome $\mathrm{c}$ from mitochondria: a primary site for Bcl-2 regulation of apoptosis. Science 275: 1132-1136, 1997.

30. Hussain AR, Al-Rasheed M, Manogaran PS, Platanias LK and Kuddin S: Curcumin induces apoptosis via inhibition of PI3'kinase/AKT pathway in acute T cell leukemias. Apoptosis 11: 245-254, 2006.

31. Deeb DD, Jiang H, Gao X, Divine G, Dulchavsky SA and Gautam SC: Chemosensitization of hormone-refractory prostate cancer cells by curcumin to TRAIL-induced apoptosis. J Exp Ther Oncol 5: 81-91, 2005.

32. Nakamura Y, Kawakami M, Yoshihiro A, Miyoshi N, Ohigashi H, Kawai K, Osawa $\mathrm{T}$ and Uchida K: Involvement of the mitochondrial death pathway in chemopreventive benzyl isothiocyanate-induced apoptosis. J Biol Chem 277: 8492-8499, 2002.

33. Hail NJ, Konopleva M, Sporn M, Lotan R and Andreeff M: Evidence supporting a role for calcium in apoptosis induction by the synthetic triterpenoid 2-cyano-3,12-dioxooleana-1,9dien-28-oic acid (CDDO). J Biol Chem 279: 11179-11187, 2004.

34. Green DR and Reed JC: Mitochondria and apoptosis. Science 281: 1309-1312, 1998

35. Uddin S, Hussain AR, Manogaran PS, Al-Hussein K, Platanias LC, Gutierrez MI and Bhatia KG: Curcumin suppresses growth and induces apoptosis in primary effusion lymphoma. Oncogene 24: 7022-7030, 2005.

36. Liao YF, Hung HC, Hour TC, Hsu PC, Kao MC, Tsay GJ and Liu GY: Curcumin induces apoptosis through an ornithine decarboxylase-dependent pathway in human promyelocytic leukemia HL-60 cells. Life Sci 82: 367-375, 2008. 